\title{
Multiparametric magnetic resonance imaging- transrectal ultrasound fusion biopsies increase the rate of cancer detection in populations with a low incidence of prostate cancer
}

\author{
Rohit Kaushal ${ }^{1}$, Chandan J. Das ${ }^{2}$, Prabhjot Singh ${ }^{1}$, Prem Nath Dogra ${ }^{1}$, Rajeev Kumar $^{1}$ \\ Departments of 'Urology and ${ }^{2}$ Radiodiagnosis, All India Institute of Medical Sciences, New Delhi, India
}

Purpose: To prospectively evaluate the diagnostic yield of multiparametric magnetic resonance imaging (mpMRl)-fusion, transrectal ultrasound (TRUS)-guided prostate biopsies for detection of prostate cancer in an Asian population with a low incidence of prostate cancer.

Materials and Methods: A total of 131 males with suspected prostate cancer were recruited to undergo fusion biopsy with the Artemis prostate fusion biopsy device (Eigen, Grass Valley, CA, USA). All patients underwent standard 12-core systematic biopsies in addition to biopsies targeted at the mpMRI-identified abnormal regions. Yield from the standard cores was compared with that from the targeted cores. Gleason scores of $4+3$ or higher were considered significant.

Results: The mean age of the patients was $63.54 \pm 7.96$ years and the mean prostate-specific antigen value was $9.75 \pm 5.35 \mathrm{ng} / \mathrm{mL}$. A total of 36 patients had cancer, of which $3(8.3 \%)$ were detected only on standard cores and $3(8.3 \%)$ only on targeted cores. Of the clinically significant cancers $(n=30)$, targeted biopsy detected a higher number $(28 / 30,93.3 \%)$ than standard biopsy $(21 / 30$, $70.0 \%)$. A total of 6 of 8 cancers (75.0\%) that were insignificant on standard biopsy were upgraded to significant cancer on targeted cores.

Conclusions: Eight percent of cancers were detected only on MRI-TRUS fusion-targeted biopsies, whereas the method upgraded more than two-thirds of insignificant cancers to significant cancers. Fusion biopsies thus provide incremental information over standard TRUS biopsies in the diagnosis of significant prostate cancer in populations with a low incidence of prostate cancer.

Keywords: Biopsy; Magnetic resonance imaging; Prostatic neoplasms

This is an Open Access article distributed under the terms of the Creative Commons Attribution Non-Commercial License (http://creativecommons.org/licenses/by-nc/4.0) which permits unrestricted non-commercial use, distribution, and reproduction in any medium, provided the original work is properly cited.

\section{INTRODUCTION}

Transrectal ultrasound (TRUS)-guided systematic biopsies of the prostate do not pick up all prostate cancer and many lesions may be missed [1-4]. Multiparametric magnetic resonance imaging (mpMRI) is among the most common imaging modalities used to aid detection of prostate cancer [3,4]. mpMRI involves one anatomical sequence (T2-weighted)

Received: 20 November, 2018 - Accepted: 11 February, 2019

Corresponding Author: Rajeev Kumar

Department of Urology, All India Institute of Medical Sciences, Ansari Nagar, New Delhi 110029, India

TEL: +91-1126594884, FAX: +91-1126588663, E-mail: rajeev.urology@aiims.edu

ORCID: https://orcid.org/0000-0002-0783-1101 
with at least one functional sequence such as diffusionweighted imaging (DWI), dynamic contrast enhancement (DCE), or spectroscopy (MRS) and is also used in the current standard Prostate Imaging-Reporting and Data System (PIRADS) classification for prostate lesions [5]. Lesions seen on mpMRI correlate with actual tumor location on radical prostatectomy specimens, and the level of suspicion on imaging correlates with the D'Amico stratification [6,7].

Lesions identified on mpMRI can be targeted for biopsy either within the MRI machine (in-bore) or during a TRUS biopsy using either cognitive localization or devices that 'fuse' MRI images with real-time ultrasound. Cognitive biopsy relies on operator expertise, whereas inbore biopsy is expensive, time-consuming, and uncomfortable for the patient, and has limited availability. While MRITRUS fusion biopsies offer an opportunity to harness the advantage of mpMRI data, their value in routine clinical practice is not standard [8]. Asian populations have a lower incidence of prostate cancer than reported in Western nations [9]. Since the sensitivity of a test can vary according to the prevalence of disease, it is possible that the performance of fusion biopsies would differ in these populations [10].

We previously reported our experience with cognitive fusion biopsies using MRS data and found that targeting improves detection in our population where the biopsy positivity rate is otherwise low [11,12]. Since cognitive fusion and MRS are difficult techniques to perform, we assessed the role of an MRI-TRUS fusion device in increasing the yield of biopsies in patients with serum prostate-specific antigen (PSA) values between 4 and $20 \mathrm{ng} / \mathrm{mL}$ and those with prior negative results on standard 12-core TRUS biopsies.

\section{MATERIALS AND METHODS}

\section{Setting}

This prospective study was performed at a single tertiary referral center in a country (India) with a low incidence of prostate cancer over an 18-month period beginning in August 2015. The study was approved by the Institutional Ethics Committee, All India Institute of Medical Sciences, Ansari Nagar, New Delhi (approval number: IECPG-526) and conformed to the provisions of the Declaration of Helsinki, and all subjects provided written informed consent.

\section{Patients}

All patients with clinical indications for prostate biopsy (total PSA between 4 and $20 \mathrm{ng} / \mathrm{mL}$ or an abnormal result on a digital rectal examination [DRE] suspicious for prostate cancer) were screened for inclusion. Patients in whom MRI was contraindicated or not feasible, those who refused to participate in the study, and those with diffuse hard nodular prostates were excluded. Patients in whom no abnormalities were found by MRI were also excluded from the study.

\section{Procedural details}

All patients underwent mpMRI of the prostate (Philips Ingenia 3T Machine; Philips, Amsterdam, The Netherlands) before the biopsy. All MRI results were reported by a single radiologist using the PI-RADS classification (version II) [5]. Identifiable abnormal lesions were marked on mpMRI using the PROFUSE software (Eigen, Grass Valley, CA, USA) (Fig. 1) and targeted for biopsy on the Artemis MRI-TRUS fusion device (Eigen). A standard systematic 12-core biopsy (base, mid-gland, and apex; lateral and paramedian; both lobes) preceded targeted biopsies from mpMRI-identified lesions. At least one and preferably two cores were taken from each of the targeted lesions. All biopsies were individually labeled and processed.

All patients received a preprocedural prophylactic antibiotic and enema. All biopsies were performed on an outpatient basis with infiltrative local anaesthesia on either side at the base of prostate. The whole gland was scanned over 360 degrees, after which the image was registered and then segmented and refined in both transverse and sagittal dimensions by the software. The refined image was then fused to the premarked mpMRI images that had already been uploaded on the device. For each core sampled, the needle track was automatically saved by the software. At the end of the procedure, a PDF file was generated for each patient showing the three-dimensional track of the sampled cores (Fig. 2). Complications were noted before discharge, during follow-up if the patient self-reported, and with direct

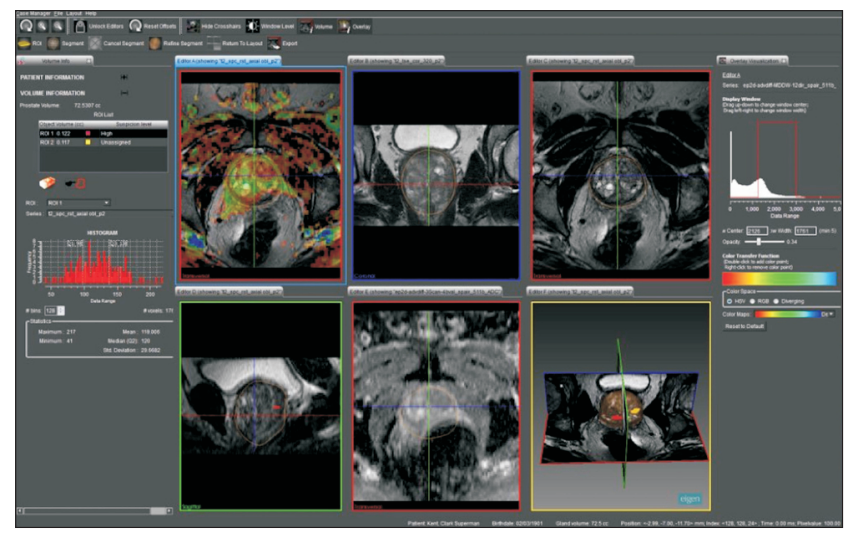

Fig. 1. PROFUSE software (Eigen, Grass Valley, CA, USA) for assignment of region of interest. 

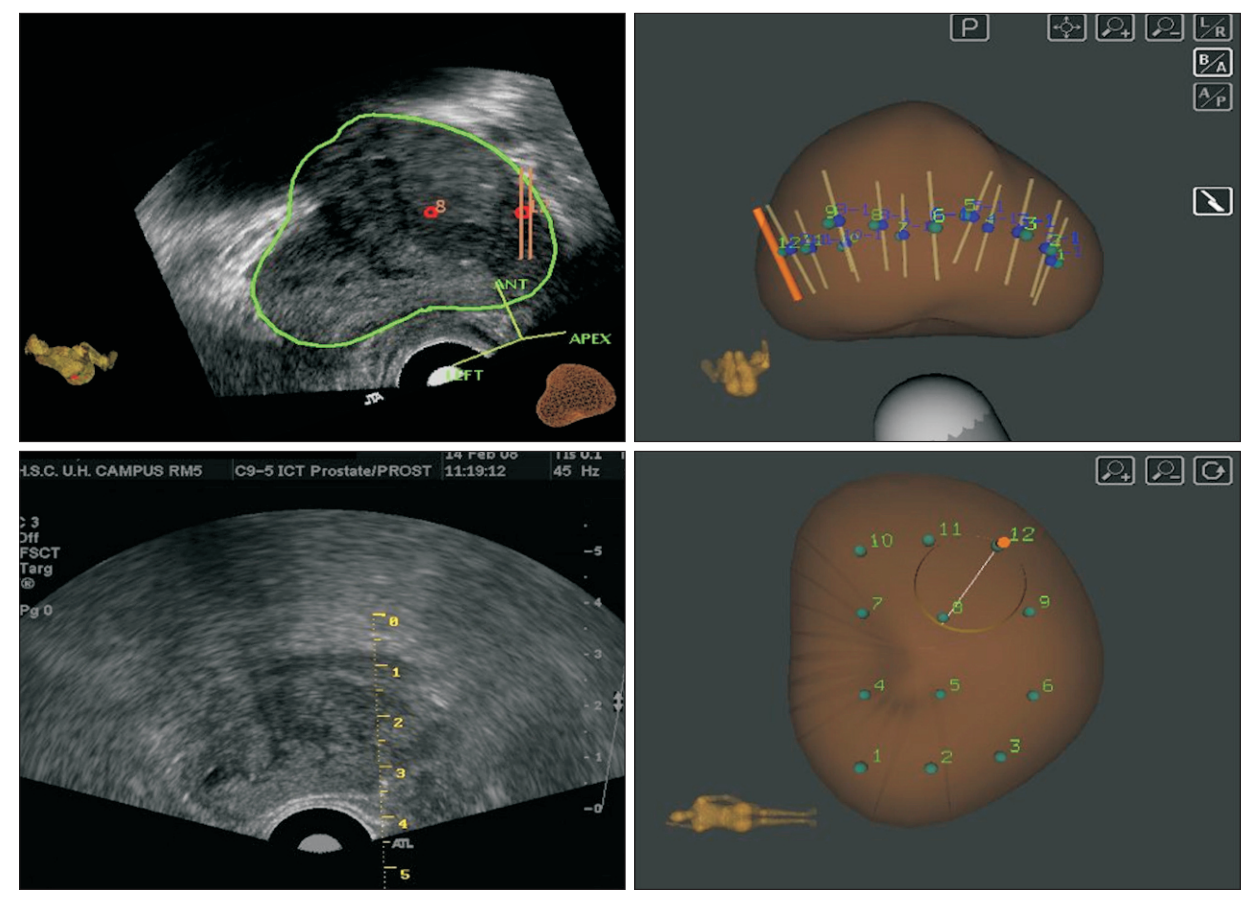

Fig. 2. Needle tracking of each core biopsied. enquiry at the next visit.

\section{Interpretation}

The rate of cancer detection was compared between systematic and targeted biopsies. Additionally, change in Gleason scoring and change from nonsignificant to significant cancer was compared on the basis of each of the two biopsy modalities. Cancers with a Gleason's score (GS) of $3+3$ or $3+4$ were considered clinically insignificant cancers, whereas those with GS $4+3$ or higher were considered clinically significant [13,14]. Additional analyses were performed for patients who had a prior negative biopsy result.

\section{Statistical analysis}

The data were analyzed by using STATA statistical software (version 14.0). Quantitative data were expressed as mean, standard deviation, and median (minimum-maximum) as appropriate. Qualitative data were expressed as frequency and percentage. Fisher's exact test was used to check the association between the variables. Student's t-test and Mann Whitney U test were used to compare normal and skewed variables, respectively. The $p$-values $\leq 0.05$ were considered as statistically significant.

\section{RESULTS}

During the study period, 210 patients were screened, of whom 131 were included in the study. Their mean age was
63.5 years and their mean serum PSA value was $9.75 \pm 5.35$ $\mathrm{ng} / \mathrm{mL}$. Mean prostate volume was $54.2 \pm 30.9 \mathrm{~cm}^{3}$. A total of 30 patients (22.9\%) had a suspicious nodule on a DRE.

The PI-RADS score on mpMRI ranged from 2 to 5 with a median of 3 . A median of 14 cores (range, 13-20 cores) were biopsied in each patient, of which the median number of targeted cores was 2 (range, 1-8 cores) (Table 1). Malignancy on biopsy was detected in 36 patients $(27.5 \%)$. In three patients, cancer was detected exclusively on systematic biopsy, whereas in another three cancer was detected exclusively in targeted cores. The detection rate with each modality was thus $91.7 \%$ (33/36). Thirty of the cancers were significant (83.3\%). Of these, 21 (70.0\%) were picked up by systematic biopsies and the targeted biopsies picked up 28 (93.3\%) (Table 1).

Among the 30 patients who had cancer detected on both systematic and targeted biopsies, 8 (26.7\%) had discordant a GS between systematic and targeted biopsies and were upgraded on targeted cores (Table 2). Of these, 6 were upgraded from clinically insignificant to significant cancer.

Among patients with a prior negative biopsy result $(\mathrm{n}=17)$, five (29.4\%) were detected to have cancer on fusion biopsy. Of these, three had cancer detected only on systematic cores, and none had cancer detected exclusively on targeted cores, whereas the other two had one core each of systematic and targeted biopsies positive. Two of these five had clinically significant cancers.

Eight patients (6.1\%) developed a complication, of whom five had post-biopsy fever requiring admission and paren- 
Table 1. Outcomes of biopsy

\begin{tabular}{|c|c|}
\hline Parameters & Value \\
\hline Total & 131 \\
\hline \multicolumn{2}{|l|}{ Number of cores biopsied } \\
\hline Overall & $14(13-20)$ \\
\hline Targeted & $2(1-8)$ \\
\hline \multicolumn{2}{|l|}{ Number of cores positive } \\
\hline Overall & $6.5(1-19)$ \\
\hline Systematic & $3(0-12)$ \\
\hline Targeted & $2(0-7)$ \\
\hline \multicolumn{2}{|c|}{ Highest PI-RADS score among patients with cancer (total $n=36$ ) } \\
\hline 2 & $2(5.6)$ \\
\hline 3 & $9(25.0)$ \\
\hline 4 & $8(22.2)$ \\
\hline 5 & $17(47.2)$ \\
\hline Patients with a prior negative biopsy & $17(13.0)$ \\
\hline Cancer positive on biopsy & $36(27.5)$ \\
\hline Clinically significant cancers (total $n=36$ ) & $30(83.3)$ \\
\hline Systematic cores (total $n=30$ ) & $21(70.0)$ \\
\hline Targeted cores (total $n=30$ ) & $28(93.3)$ \\
\hline Cancer detected exclusively on systematic cores & 3 \\
\hline Clinically significant & 2 \\
\hline Cancer detected exclusively on targeted cores & 3 \\
\hline Clinically significant & 3 \\
\hline Complications & $8(6.1)$ \\
\hline
\end{tabular}

Values are presented as number only, median (range), or number (\%). PI-RADS, prostate imaging reporting and data system.

teral antibiotics, one had hematuria, and two had urine retention.

\section{DISCUSSION}

In our cohort of males with historically low rates of detection of prostate cancer, mpMRI-TRUS fusion biopsies detected malignancy in $27.5 \%$ of males. A total of $83.3 \%$ of these cancers were clinically significant, and targeted biopsies identified $93.3 \%$ of these significant cancers. However, targeted biopsies alone would have missed 3 of 36 cancers, including 2 of 30 significant cancers.

The use of mpMRI targeting for prostate biopsies may serve two purposes. Apart from identifying potential targets for biopsy based on MR data, use of mpMRI may help to improve yield even from systematic cores by ensuring a more even sampling of the gland than TRUS guidance alone. Standard systematic biopsies have a low sensitivity and tend to detect more indolent cancers [15]. While there is increasing data on the use of fusion techniques, nearly all of this comes from populations with relatively high prevalences of prostate cancer [13,16-20]. Some of these studies have
Table 2. Discordant Gleason score between targeted cores versus systematic cores $(n=30)$

\begin{tabular}{ccc}
\hline \multirow{2}{*}{ Patient } & \multicolumn{2}{c}{ Gleason score } \\
\cline { 2 - 3 } & Systematic cores & Targeted cores \\
\hline 1 & $3+3$ & $4+3$ \\
2 & $3+3$ & $4+3$ \\
3 & $3+3$ & $4+3$ \\
4 & $3+3$ & $4+3$ \\
5 & $3+4$ & $4+3$ \\
6 & $3+4$ & $4+3$ \\
7 & $4+3$ & $5+4$ \\
8 & $4+4$ & $5+4$ \\
\hline
\end{tabular}

reported cancer detection rates, with fusion techniques, as high as $89 \%$ [16]. The prevalence of cancer in India and other Asian countries is much lower, at times less than $5.6 \%$ of the rates in other populations $(7 / 100,000$ versus $126 / 100,000)$ $[9,21,22]$. Disease prevalence affects the performance of a diagnostic test and may cause a change in its sensitivity or specificity of up to $40 \%$ [10]. Cancer detection rates among our population of men tend be low, with reported detection rates of $13 \%$ for men with PSA below $20 \mathrm{ng} / \mathrm{mL}$ [21]. Our own data from a similar population of patients found a low $10.1 \%$ cancer detection rate on standard TRUS-guided biopsies, although one of the confounders in that cohort may have been the lower average PSA [11]. The current study found a higher yield not only on targeted cores but also on the systematic cores. This would suggest a role of fusion techniques in increasing the yield even on systematic biopsies. Similar data have previously been reported using fusion techniques in these populations which otherwise have a low incidence of prostate cancer [23]. Such variations make it important that data on performance of investigations be assessed in different populations, because direct translation of information from one study may not be possible.

The PI-RADS classification has simplified mpMRI reporting and allows uniformity for comparisons [24]. Similar to previously reported data, our findings showed that a greater proportion of positive biopsies corresponded to higher PI-RADS scores [16]. However, cancer was found even on lesions reported as PI-RADS-2, which suggests that when a fusion biopsy is planned, PI-RADS-2 lesions should also be targeted.

mpMRI-based targeted biopsies have been reported to result in higher cancer detection rates along with detection of more clinically significant cancers $[17,18,25]$. We found that targeted cores detected a high $93.3 \%$ of all significant cancers in our cohort. However, targeted biopsies failed to detect three cancers, including two significant ones, 
suggesting that the systematic cores should not be omitted from the biopsy protocol. On the contrary, performing systematic biopsies alone would not only have missed three cancers altogether (all significant cancers), but would have understaged six additional cases. Overall, 9 of 30 significant cancers (30.0\%) would have been missed by systematic biopsies alone. Similar studies have shown upgrading rates of $32 \%$ to $35 \%$ [13]. Our data are consistent with the findings of the prospective PROFUS trial that evaluated targeted biopsies [19]. That study reported a cancer detection rate of $32 \%$ with the primary benefit of fusion being the detection of higher grade cancers.

Our study was limited by the lack of a control arm including patients who did not undergo nonfusion biopsies. However, our previously reported data [11,12], and that of other reports from this region [21,23], suggest that the cancer detection rate of systematic TRUS biopsies is low in our patients. Our current results can thus be compared to such controls. We were also limited by the lack of radical prostatectomy step-section histology results for comparison. However, since the primary objective was to evaluate the rate of cancer detection on biopsy, the objective was fulfilled.

\section{CONCLUSIONS}

mpMRI-TRUS fusion targeted biopsies improved the detection of clinically significant cancers in comparison with systematic TRUS-guided biopsies. However, targeted biopsies alone would have missed $8.3 \%$ of cancers, including $6.7 \%$ of significant cancers. The use of fusion techniques increased the overall cancer detection rate in comparison with historical systematic TRUS biopsies in our population with a low incidence of prostate cancer, which suggests a potential role for this technique.

\section{CONFLICTS OF INTEREST}

The authors have nothing to disclose.

\section{REFERENCES}

1. Babaian RJ, Toi A, Kamoi K, Troncoso P, Sweet J, Evans R, et al. A comparative analysis of sextant and an extended 11-core multisite directed biopsy strategy. J Urol 2000;163:152-7.

2. Ouzzane A, Puech P, Lemaitre L, Leroy X, Nevoux P, Betrouni $\mathrm{N}$, et al. Combined multiparametric MRI and targeted biopsies improve anterior prostate cancer detection, staging, and grading. Urology 2011;78:1356-62.

3. Turkbey B, Pinto PA, Mani H, Bernardo M, Pang Y, McKinney
YL, et al. Prostate cancer: value of multiparametric MR imaging at $3 \mathrm{~T}$ for detection--histopathologic correlation. Radiology 2010;255:89-99.

4. Puech P, Potiron E, Lemaitre L, Leroy X, Haber GP, Crouzet S, et al. Dynamic contrast-enhanced-magnetic resonance imaging evaluation of intraprostatic prostate cancer: correlation with radical prostatectomy specimens. Urology 2009;74:10949.

5. Seo JW, Shin SJ, Taik Oh Y, Jung DC, Cho NH, Choi YD, et al. PI-RADS version 2: detection of clinically significant cancer in patients with biopsy gleason score 6 prostate cancer. AJR Am J Roentgenol 2017;209:W1-9.

6. Turkbey B, Mani H, Shah V, Rastinehad AR, Bernardo M, Pohida T, et al. Multiparametric 3T prostate magnetic resonance imaging to detect cancer: histopathological correlation using prostatectomy specimens processed in customized magnetic resonance imaging based molds. J Urol 2011;186:1818-24.

7. Rastinehad AR, Baccala AA Jr, Chung PH, Proano JM, Kruecker J, Xu S, et al. D'Amico risk stratification correlates with degree of suspicion of prostate cancer on multiparametric magnetic resonance imaging. J Urol 2011;185:815-20.

8. Jain S, Saxena S, Kumar A. Epidemiology of prostate cancer in India. Meta Gene 2014;2:596-605.

9. Ito K. Prostate cancer in Asian men. Nat Rev Urol 2014;11:197212.

10. Leeflang MM, Rutjes AW, Reitsma JB, Hooft L, Bossuyt PM. Variation of a test's sensitivity and specificity with disease prevalence. CMAJ 2013;185:E537-44.

11. Javali TD, Dwivedi DK, Kumar R, Jagannathan NR, Thulkar S, Dinda AK. Magnetic resonance spectroscopy imaging-directed transrectal ultrasound biopsy increases prostate cancer detection in men with prostate-specific antigen between $4-10 \mathrm{ng} / \mathrm{mL}$ and normal digital rectal examination. Int J Urol 2014;21:25762.

12. Kumar R, Nayyar R, Kumar V, Gupta NP, Hemal AK, Jagannathan NR, et al. Potential of magnetic resonance spectroscopic imaging in predicting absence of prostate cancer in men with serum prostate-specific antigen between 4 and $10 \mathrm{ng} / \mathrm{ml}$ : a follow-up study. Urology 2008;72:859-63.

13. Siddiqui MM, Rais-Bahrami S, Truong H, Stamatakis L, Vourganti S, Nix J, et al. Magnetic resonance imaging/ultrasoundfusion biopsy significantly upgrades prostate cancer versus systematic 12-core transrectal ultrasound biopsy. Eur Urol 2013;64:713-9.

14. Moore CM, Kasivisvanathan V, Eggener S, Emberton M, Fütterer JJ, Gill IS, et al.; START Consortium. Standards of reporting for MRI-targeted biopsy studies (START) of the prostate: recommendations from an International Working Group. Eur Urol 2013;64:544-52. 
15. Heidenreich A, Bellmunt J, Bolla M, Joniau S, Mason M, Matveev V, et al. EAU guidelines on prostate cancer. Part 1: screening, diagnosis, and treatment of clinically localised disease. Eur Urol 2011;59:61-71.

16. Pinto PA, Chung PH, Rastinehad AR, Baccala AA Jr, Kruecker J, Benjamin CJ, et al. Magnetic resonance imaging/ultrasound fusion guided prostate biopsy improves cancer detection following transrectal ultrasound biopsy and correlates with multiparametric magnetic resonance imaging. J Urol 2011;186:12815.

17. Vourganti S, Rastinehad A, Yerram N, Nix J, Volkin D, Hoang A, et al. Multiparametric magnetic resonance imaging and ultrasound fusion biopsy detect prostate cancer in patients with prior negative transrectal ultrasound biopsies. J Urol 2012;188:2152-7.

18. Sonn GA, Chang E, Natarajan S, Margolis DJ, Macairan M, Lieu $\mathrm{P}$, et al. Value of targeted prostate biopsy using magnetic resonance-ultrasound fusion in men with prior negative biopsy and elevated prostate-specific antigen. Eur Urol 2014;65:80915.

19. Wysock JS, Rosenkrantz AB, Huang WC, Stifelman MD, Lepor $\mathrm{H}$, Deng FM, et al. A prospective, blinded comparison of magnetic resonance (MR) imaging-ultrasound fusion and visual estimation in the performance of MR-targeted prostate biopsy: the PROFUS trial. Eur Urol 2014;66:343-51.
20. Baco E, Rud E, Eri LM, Moen G, Vlatkovic L, Svindland A, et al. A randomized controlled trial to assess and compare the outcomes of two-core prostate biopsy guided by fused magnetic resonance and transrectal ultrasound images and traditional 12-core systematic biopsy. Eur Urol 2016;69:149-56.

21. Patil SR, Pawar PW, Sawant AS, Patil AV, Narwade SS, Mundhe ST, et al. TRUS biopsy yield in Indian population: a retrospective analysis. J Clin Diagn Res 2017;11:PC01-5.

22. Jemal A, Ward EM, Johnson CJ, Cronin KA, Ma J, Ryerson B, et al. Annual report to the nation on the status of cancer, 19752014, featuring survival. J Natl Cancer Inst 2017;109.

23. Bansal S, Gupta NP, Yadav R, Khera R, Ahlawat K, Gautam D, et al. Multiparametric magnetic resonance imaging-transrectal ultrasound fusion prostate biopsy: a prospective, single centre study. Indian J Urol 2017;33:134-9.

24. Barentsz JO, Richenberg J, Clements R, Choyke P, Verma S, Villeirs G, et al. ESUR prostate MR guidelines 2012. Eur Radiol 2012;22:746-57.

25. Hoeks CM, Schouten MG, Bomers JG, Hoogendoorn SP, Hulsbergen-van de Kaa CA, Hambrock T, et al. Three-Tesla magnetic resonance-guided prostate biopsy in men with increased prostate-specific antigen and repeated, negative, random, systematic, transrectal ultrasound biopsies: detection of clinically significant prostate cancers. Eur Urol 2012;62:902-9. 